Open Access

\title{
Detection of elevated right ventricular extracellular volume in pulmonary hypertension using Accelerated and Navigator-Gated Look-Locker Imaging for Cardiac T1 Estimation (ANGIE) cardiovascular magnetic resonance
}

\author{
Bhairav B. Mehta², Daniel A. Auger², Jorge A. Gonzalez', Virginia Workman¹, Xiao Chen², Kelvin Chow², \\ Claire J. Stump², Sula Mazimba', Jamie L. W. Kennedy', Elizabeth Gay', Michael Salerno 1,2,3, \\ Christopher M. Kramer ${ }^{1,3}$, Frederick H. Epstein ${ }^{2,3}$ and Kenneth C. Bilchick ${ }^{1 *}$
}

\begin{abstract}
Background: Assessment of diffuse right ventricular (RV) fibrosis is of particular interest in pulmonary hypertension (PH) and heart failure (HF). Current cardiovascular magnetic resonance (CMR) T1 mapping techniques such as Modified Look-Locker inversion recovery (MOLLI) imaging have limited resolution, but accelerated and navigator-gated Look-Locker imaging for cardiac T1 estimation (ANGIE) is a novel CMR sequence with spatial resolution suitable for T1 mapping of the RV. We tested the hypothesis that patients with PH would have significantly more RV fibrosis detected with MRI ANGIE compared with normal volunteers and patients having HF with reduced (LV) ejection fraction (HFrEF) without co-existing PH, independent of RV dilatation and dysfunction.
\end{abstract}

Methods: Patients with World Health Organization group 1 or group $4 \mathrm{PH}$, patients with HFrEF without PH, and normal volunteers were recruited to undergo contrast-enhanced CMR. RV and LV extracellular volume fractions (RV-ECV and LV-ECV) were determined using pre-contrast and post-contrast T1 mapping using ANGIE (RV and LV) and MOLLI (LV only).

Results: Thirty-two participants (53.1\% female, median age 52 years, IQR 26-65 years) were enrolled, including $n=12$ with $\mathrm{PH}, n=10$ having HFrEF without co-existing PH, and $n=10$ normal volunteers. ANGIE ECV imaging was of high quality, and ANGIE measurements of LV-ECV were highly correlated with those of MOLLI $(r=0.91 ; p<0.001)$. The RV-ECV in PH patients was $27.2 \%$ greater than the RV-ECV in normal volunteers $(0.341$ v. $0.268 ; p<0.0001)$ and $18.9 \%$ greater than the RV-ECV in HFrEF patients without PH (0.341 v. 0.287; $p<0.0001)$. RV-ECV was greater than LV-ECV in $\mathrm{PH}(\mathrm{RV}$-LV difference $=0.04$ ), but RV-ECV was nearly equivalent to LV-ECV in normal volunteers (RV-LV difference $=0.002$ ) $(p<0.0001$ for RV-LV difference in PH versus normal volunteers). RV-ECV was linearly associated with both increasing RVEDVI $(p=0.049)$ and decreasing RVEF $(p=0.04)$ in a multivariable linear model, but PH was still associated with greater RV-ECV even after adjustment for RVEDVI and RVEF.

* Correspondence: kcb7f@hscmail.mcc.virginia.edu

'Department of Medicine, University of Virginia Health System, P.O. Box 800158, Charlottesville, VA 22908, USA

Full list of author information is available at the end of the article

\section{Biomed Central}

(c) 2015 Mehta et al. Open Access This article is distributed under the terms of the Creative Commons Attribution 4.0 International License (http://creativecommons.org/licenses/by/4.0/), which permits unrestricted use, distribution, and reproduction in any medium, provided you give appropriate credit to the original author(s) and the source, provide a link to the Creative Commons license, and indicate if changes were made. The Creative Commons Public Domain Dedication waiver (http://creativecommons.org/publicdomain/zero/1.0/) applies to the data made available in this article, unless otherwise stated. 
(Continued from previous page)

Conclusions: Pre- and post-contrast ANGIE imaging provides high-resolution ECV determination for the RV. PH is independently associated with increased RV-ECV even after adjustment for RV dilatation and dysfunction, consistent with an independent effect of PH on fibrosis. ANGIE RV imaging merits further clinical evaluation in $\mathrm{PH}$.

Keywords: Magnetic resonance, Pulmonary hypertension, Fibrosis, T1 mapping, Extracellular volume fraction, Right ventricle

\section{Background}

Right ventricular (RV) dysfunction in pulmonary hypertension $(\mathrm{PH})$ is not only an indicator of severity of disease but also a cause of heart failure (HF) [1,2] and the most important predictor of survival [3-6]. RV pathophysiology in $\mathrm{PH}$ and $\mathrm{HF}$ involves complex interactions among myocardial injury, altered gene expression, ventricular remodeling, the renin-angiotensin-aldosterone system, natriuretic peptides, the endothelin system, and various cytokines, leading to fibrosis, cell death, myocardial dysfunction, and reduced systemic perfusion [7-9]. Left ventricular (LV) fibrosis is of considerable interest and readily evaluated in hypertension and other cardiovascular diseases [10], while RV fibrosis is more difficult to assess than LV fibrosis because of the specific attributes of RV anatomy, including the thin RV free wall, differences in structure between the septum and free wall, and the complex 3D structure including an outflow tract. Although echocardiography provides some information on RV structure and function [11], cardiovascular magnetic resonance (CMR) is generally considered the gold standard in this regard, even though CMR assessment of fibrosis in $\mathrm{PH}$ has been limited to evaluation of scar by late gadolinium enhancement (LGE) at LV septal insertion sites $[12,13]$. Consequently, the strength of CMR for the RV has traditionally been high-definition cine imaging with a steady-state free precession protocol rather than contrast-enhanced fibrosis imaging.

There is a growing literature for T1 mapping of the LV, and the myocardial extracellular volume (ECV) fraction determined with this technique has been shown to correlate with histologic fibrosis [14-22]; however, current T1 mapping methods have insufficient spatial resolution for effective RV fibrosis imaging [14]. For example, limitations of common T1 mapping methods such as modified LookLocker inversion recovery imaging (MOLLI) [14] include suboptimal spatial resolution and the need for breath holding during scanning. In response, our group has developed a method for high quality CMR T1 mapping of the RV called accelerated and navigator-gated Look-Locker imaging for cardiac T1 estimation (ANGIE) [20], which employs navigator gating and compressed sensing to provide a 4-fold improvement in spatial resolution compared with MOLLI without the need for a breath hold.
Considering that we have previously demonstrated the accuracy of ANGIE for native T1 mapping of the LV and RV [20], the aim of the present study was to demonstrate the feasibility and effectiveness of contrastenhanced ANGIE imaging for determination of RV-ECV and LV-ECV in a cross-sectional study composed of a) patients with World Health Organization (WHO) groups 1 or $4 \mathrm{PH}$ (without LV systolic dysfunction), b) patients having $\mathrm{HF}$ with reduced (LV) ejection fraction (LVEF) (HFrEF) without co-existing $\mathrm{PH}$, and c) normal volunteers. Because the RV in $\mathrm{PH}$ is compromised by high pressures, which lead to chamber dilatation, dysfunction, and fibrosis, we hypothesized that patients with $\mathrm{PH}$ would have significantly more RV fibrosis detected with ANGIE compared with normal volunteers and patients having HF with reduced (LV) ejection fraction without co-existing $\mathrm{PH}$, independent of RV dilatation and dysfunction.

\section{Methods}

\section{Patient cohort}

All participants provided written informed consent, and the study was conducted according to standard ethical principles, as documented in the study protocol approved by our Institutional Review Board for Human Subjects Research. Patients were recruited to undergo CMR with a gadolinium-based contrast agent. The study population was divided into three groups of subjects. The PH cohort $(n=12)$ consisted of patients diagnosed with PH classified as WHO group 1 (pulmonary arterial hypertension) or 4 (chronic thromboembolic $\mathrm{PH}$ ). WHO groups 1 and 4 were chosen for the $\mathrm{PH}$ cohort rather than WHO groups 2 ( $\mathrm{PH}$ due to left heart diseases), 3 ( $\mathrm{PH}$ due to lung diseases and/or hypoxemia), or 5 ( $\mathrm{PH}$ with unclear multifactorial mechanisms) in order to provide a $\mathrm{PH}$ cohort with preserved LV systolic function without extensive comorbid cardiopulmonary disease. The second group consisted of patients with HFrEF without PH $(n=10)$. The third group $(n=10)$ consisted of healthy volunteers who served as the control group for this study. Patients were excluded from the study if they had standard contraindications for gadolinium-enhanced CMR. All patients were required to have a glomerular filtration rate (GFR) of at least $45 \mathrm{cc} /$ minute $/ 1.73 \mathrm{~m}^{2}$ (calculated based on a serum creatinine drawn within 30 days 
of the CMR study using the Modification of Diet in Renal Disease [MDRD] equation). Although we did not enroll patients on intravenous prostacyclin, these patients could be included in future studies, but would require nursing assistance with the tubing extension to maintain a connection with the pump during the scan.

\section{CMR protocol and image reconstruction}

All CMR studies were performed using a $1.5 \mathrm{~T}$ MRI scanner (Avanto, Siemens, Erlangen, Germany). Peripheral intravenous access was obtained, and the hematocrit was determined from a peripheral blood draw for the calculation of ECV, as described in more detail below. After electrocardiography leads were placed, each patient was positioned in the magnet. A 5-channel phased-array radiofrequency coil was used for signal reception. With respect to the CMR imaging protocol for this study, a standard localizer was first performed to identify the cardiac short- and long-axis planes. Steady-state free precession cine images were then obtained for assessment of the RV end-diastolic volume index (RVEDVI), the LV enddiastolic volume index (LVEDVI), RV end-systolic volume index (RVESVI), LV end-systolic volume index (LVESVI), RVEF, and LVEF. Specific parameters included: $\mathrm{TR}=2.7 \mathrm{~ms}, \mathrm{TE}=1.3 \mathrm{~ms}$, flip angle $=70^{\circ}$, FOV $=300-350 \mathrm{~mm}$, and in-plane spatial resolution = $1.8 \times 1.4 \mathrm{~mm}^{2}$. The entire heart was covered using a stack of short-axis slices with a thickness of $8 \mathrm{~mm}$ and an inter-slice gap of $2 \mathrm{~mm}$. Three long-axis slices were also acquired in two-chamber, three-chamber, and four-chamber views.

Next, pre-contrast ANGIE T1 mapping was performed. For ANGIE, two mid-ventricular short-axis slices were imaged at end systole to maximize RV wall thickness and separation of the RV wall from the liver and the chest wall during imaging. The midventricular short-axis slices were aligned perpendicular to the major-axis of the RV and positioned in a region with minimal variation along the through plane direction in order to minimize the partial volume effects. The ANGIE protocol utilized repetitions of an inversion pulse followed by four consecutive ECG-triggered data acquisitions and a recovery period of two $R-R$ intervals, as well as the following sequence parameters: $\mathrm{TR}=3.2 \mathrm{~ms}, \quad \mathrm{TE}=1.6 \mathrm{~ms}$, flip angle $=35^{\circ}, \quad \mathrm{FOV}=$ $270-340 \mathrm{~mm}$, matrix size $=224 \times 224$, in-plane resolution $=1.2-1.4 \times 1.2-1.4 \mathrm{~mm}^{2}$, slice thickness $=4 \mathrm{~mm}$, acquisition window duration $=102 \mathrm{~ms}$ per heartbeat, phase encodes per heartbeat $=32$, navigator acceptance window $= \pm 3 \mathrm{~mm}$, initial inversion time $=160 \mathrm{~ms}$, inversion time increment $=80 \mathrm{~ms}$, and acceleration rate $=2$. For all ANGIE scans, the raw $k$-space data were exported from the scanner, and image reconstruction was performed offline on a personal computer as previously described [20].

Following pre-contrast ANGIE, gadolinium-DTPA (Magnevist, Bayer Healthcare) was injected intravenously with a dose of $0.15 \mathrm{mmol} / \mathrm{kg}$. LGE CMR was performed $10 \mathrm{~min}$ following gadolinium-DTPA injection at slice locations matching those acquired using cine CMR. A phase sensitive inversion recovery sequence was used with the inversion time set to null normal appearing myocardium. Specific sequence parameters included $\mathrm{TR}=3.2 \mathrm{~ms}, \mathrm{TE}=1.6 \mathrm{~ms}$, flip angle $=25^{\circ}$, FOV $=300-340 \mathrm{~mm}$, resolution $=1.8 \times 1.3 \mathrm{~mm}^{2}$, and slice thickness $=4 \mathrm{~mm}$. The image acquisition was timed to occur at end systole for alignment with T1 mapping acquisitions. Post-contrast ANGIE T1 mapping acquisitions were performed at $20 \mathrm{~min}$ and $30 \mathrm{~min}$ after contrast administration using the same parameters and slice locations as the pre-contrast ANGIE T1 mapping acquisitions.

Modified Look-Locker inversion recovery (MOLLI) T1 mapping scans were also performed both pre- and post-contrast in $\mathrm{PH}$ patients for validation of ANGIE LV-ECV measurements. For both pre- and postcontrast imaging, we used a shorter variant of MOLLI (MOLLI 5-(R4)-3) comprised of two inversion recovery based Look-Locker experiments [23]. The shorter MOLLI sequence acquired 5 images after the first inversion, used a four heart-beat pause, and then acquired three images after the second inversion. The slice locations matched those of the ANGIE acquisition, and the image acquisition was timed to occur at end systole for alignment with the ANGIE T1 maps. Specific imaging parameters for MOLLI were $T R=$ $2.7 \mathrm{~ms}, \mathrm{TE}=1.16 \mathrm{~ms}, \quad \mathrm{FOV}=300-310 \mathrm{~mm}$, matrix size $=192 \times 154, \quad$ pixel $\quad$ size $=1.6-1.7 \times 1.9-2.1 \quad \mathrm{~mm}^{2}$, flip angle $=35^{\circ}$, slice thickness $=4 \mathrm{~mm}$, acquisition window duration $=234 \mathrm{~ms}$ per heartbeat, initial $\mathrm{TI}=$ $100 \mathrm{~ms}$, and TI increment $=80 \mathrm{~ms}$. Only LV-ECV was determined with MOLLI because MOLLI did not provide sufficient resolution for a meaningful determination of RV-ECV. In contrast, ANGIE provided sufficient resolution for the RV [Fig. 1].

\section{CMR data analysis and ECV determination}

Myocardial borders were manually delineated on steady state free precession cine images using Argus software (Siemens Medical Solutions, Munich, Germany) for determination of RVEDVI, LVEDVI, RVEF, and LVEF. All other image analysis was performed using custom software developed in MATLAB (The Mathworks, Inc., Natick, Massachusetts).

For ANGIE and MOLLI, T1 maps were computed as described previously [20]. Manual contours for the 


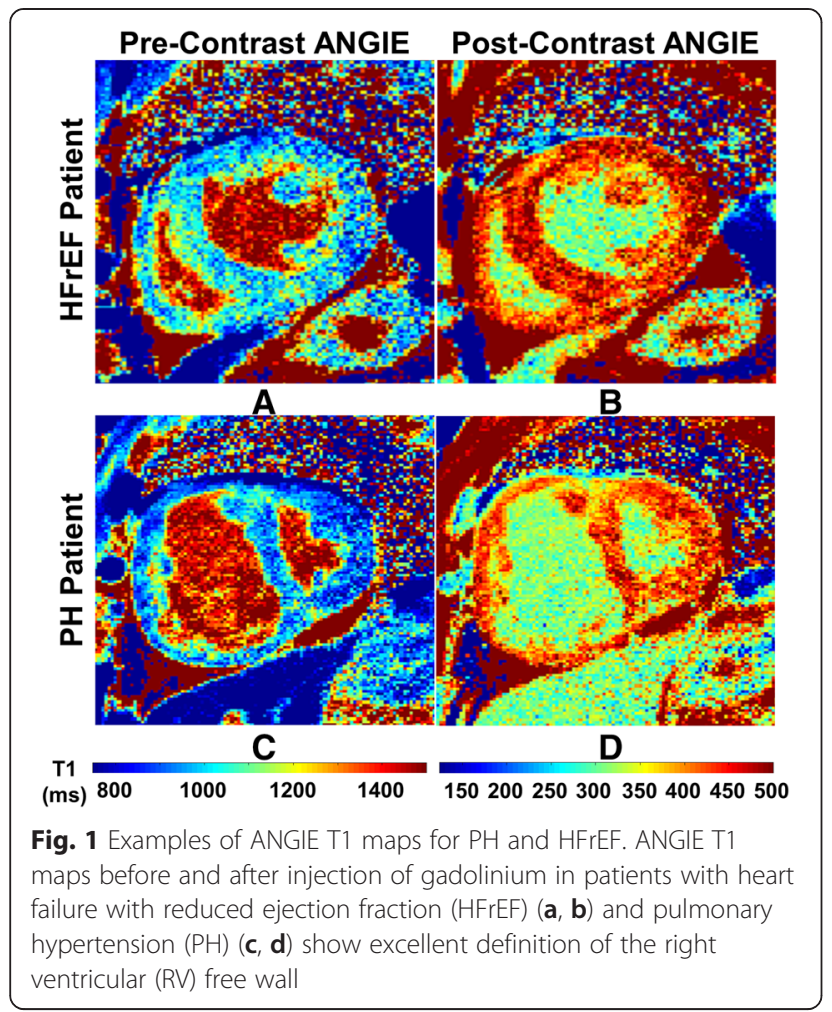

RV and LV were drawn in a conservative manner on images at one inversion time to exclude trabeculations. Non-scar regions of myocardium were identified for analysis of ECV by the absence of enhancement in the corresponding LGE image. The myocardial partition coefficient for gadolinium-DTPA $\left(\lambda_{G d}\right)$ was computed from the myocardial and the LV blood T1 estimates using the standard equation [23-25]:

$$
\begin{aligned}
& \lambda_{G d}=\frac{G d \text { concentration in myocardium }}{G d \text { concentration in blood }} \\
& =\frac{1 / T_{1 \text { myo with } G d}-1 / T_{1 \text { myo without } G d}}{1 / T_{1 \text { blood with } G d}-1 / T_{1} \text { blood without } G d}
\end{aligned}
$$

Myocardial ECV was calculated from $\lambda_{G d}$ and the blood hematocrit using the established relationship: $E C V=\lambda_{G d}(1-$ hematocrit $)$ [23-25].

\section{Assessment of $\mathrm{PH}$ with echocardiography and right heart catheterization}

Evaluation for the presence of $\mathrm{PH}$ in both groups of patients was assessed based on right heart catheterization and echocardiography results. The result obtained from the right heart catheterization was considered the gold standard. RV systolic pressures by echocardiography were assessed based on the peak tricuspid regurgitation jet velocity and right atrial pressure using the standard calculation based on the simplified Bernoulli equation.

\section{Statistical analysis}

All statistical analyses were performed using SAS 9.4 (Carey, North Carolina) and SigmaPlot 12.5 (Systat Software, Inc., San Jose, California). Continuous variables were described using the median and interquartile range. The distribution of the continuous variables between groups was assessed using the Shapiro-Wilk test for normality. For selected variables with a confirmed normal distribution, the mean was also used. Comparisons of continuous variables between groups were performed using the Kruskal-Wallis test. Categorical values were described using frequencies and percentages in the different groups, and comparisons between groups were performed using Fisher's exact test. Correlations between continuous variables were assessed using Pearson's correlation coefficient after confirmation of normality and evaluated with scatter plots. Multivariable linear regression was used to assess the complex relationship between RV-ECV, RVEF, and RVEDVI, and the underlying clinical condition.

Interobserver variability was assessed for RV-ECV, pre-contrast $\mathrm{T} 1$, and post-contrast $\mathrm{T} 1$ in 10 randomly selected participants. The intraclass correlation coefficient (ICC) is reported as the primary measure of interobserver variability.

\section{Results}

Patient cohort and baseline CMR parameters

Thirty-two participants were enrolled in the study (53.1\% female, median age 52 years old, IQR 26-65 years old). Patient characteristics for the entire cohort are described in Table 1 . Of note, $75 \%$ of $\mathrm{PH}$ patients were on advanced therapies for $\mathrm{PH}$, including $50 \%$ on an endothelin receptor antagonist (ERA), $58.3 \%$ on sildenafil, and $16.7 \%$ on a prostacyclin analog.

CMR parameters for $\mathrm{PH}$ patients, HFrEF patients, and healthy volunteers are shown in Table 2, which shows that RVEF was decreased, and both RVEDVI and RVESVI were increased in $\mathrm{PH}$ compared with HFrEF and normal volunteers $(p<0.05$ for all). Specifically, RVEF was most significantly depressed in the $\mathrm{PH}$ group (34.0\% [IQR 30.5-42.5\%]), mild-moderately depressed in the HFrEF group (41.0\% [IQR 38.0-48.0\%], and normal in volunteers (55.5\% [IQR 52.0-58.2 \%]). In HFrEF patients, LVEF was decreased $(p<0.0001)$, and LVEDVI $(p=0.0001)$, LVESVI $(p=$ $0.001)$, and LVMI $(p=0.008)$ were increased compared with $\mathrm{PH}$ patients and normal volunteers.

Right heart catheterization was used for the diagnosis of PH. Patients with HFrEF had no evidence of $\mathrm{PH}$ by 
Table 1 Baseline characteristics

\begin{tabular}{lllll}
\hline & $\mathrm{PH}(\mathrm{N}=12)$ & HFrEF $(\mathrm{N}=10)$ & Normal $(N=10)$ & $p$-value \\
\hline Age (years [IQR]) & $64.5(47.5-70.5)$ & $60.0(53.0-65.0)$ & $24.4(21.7-26.3$ & $<(80.0)$ \\
Gender (\% female) & $8(66.7)$ & $1(10.0)$ & $24.1(21.2-26.3)$ & 0.004 \\
BMI (kg/m2 [IQR]) & $25.4(23.0-29.3)$ & $29.2(26.0-32.7)$ & $0(0.0)$ & 0.47 \\
Ischemic HD (\%) & $1(8.3)$ & $8(80.0)$ & $0(0.0)$ & 0.010 .0001 \\
Congenital HD (\%) & $1^{\mathrm{a}}(8.3)$ & $1(10.0)$ & $0(0.0)$ & 1.0 \\
DM (\%) & $2(16.7)$ & $3(30.0)$ & $0(0.0)$ & 0.23 \\
OSA (\%) & $3(25.0)$ & $2(20.0)$ & $0(0.0)$ & 0.33 \\
HIV (\%) & $2(16.7)$ & $0(0.0)$ & $0(0.0)$ & 0.31 \\
CTEPH (\%) & $2(16.7)$ & $0(0.0)$ & $0(0.0)$ & 0.31 \\
Medication-ERA & $6(50.0)$ & $0(0.0)$ & $0(0.0)$ & 0.002 \\
Medication-sildenafil & $7(58.3)$ & $0(0.0)$ & $0(0.0)$ & 0.31 \\
Medication-PA & $2(16.7)$ & &
\end{tabular}

Continuous variable are reported as median (IQR)

CTEPH chronic thromboembolic pulmonary hypertension, DM diabetes mellitus, ERA endothelin receptor antagonist, HD heart disease, HIV human immunodeficiency virus, OSA obstructive sleep apnea, PA prostacyclin analogue, TE thromboembolism

${ }^{a}$ This patient has severe pulmonary hypertension resulting from late repair of a ventricular septal defect

Doppler echocardiography. RV systolic pressure was twofold higher in the $\mathrm{PH}$ cohort compared with the HFrEF cohort by Doppler echocardiography $(52.3 \mathrm{~mm}$ $\mathrm{Hg}$ versus $26.8 \mathrm{~mm} \mathrm{Hg} ;=0.01$ ).

\section{ECV mapping results}

Examples of high-resolution pre- and post-contrast ANGIE T1 maps of the LV and RV acquired from HFrEF (a-b) and PH (c-d) patients are shown in Fig. 1. These examples illustrate the high quality of ANGIE T1 maps for this cohort of patients. The ANGIE T1 maps in both patients show good definition of not only the LV but also the RV. Figure 2 illustrates determination of the RV
(A and C) and LV (B and D) myocardial partition coefficients for gadolinium-DTPA $\left(\lambda_{G d}\right)$ using T1 estimates of the myocardium and LV blood pool.

\section{Correlation of ANGIE and MOLLI LV- ECV assessments}

MOLLI acquisitions were obtained in addition to ANGIE in patients with PH $(n=12)$. The distribution of LV-ECV in these patients was determined to be normal. As shown in Fig. 3, the ANGIE measurements of LVECV in PH patients were highly correlated with MOLLI measurements $(r=0.91 ; p<0.001)$, which supports the accuracy of myocardial ECV measurement using ANGIE T1 mapping.

Table 2 CMR parameters

\begin{tabular}{|c|c|c|c|c|}
\hline & $\mathrm{PH}(N=12)$ & $\operatorname{HFrEF}(N=10)$ & Normal $(N=10)$ & $p$ value \\
\hline RVEF (\%) & $34.0(30.5-42.5)$ & $41.0(38.0-48.0)$ & $55.5(52.0-58.0)$ & 0.001 \\
\hline RVEDVI $\left(\mathrm{ml} / \mathrm{m}^{2}\right)$ & $87.9(75.0-119.2)$ & $59.0(50.4-67.9)$ & $60.8(59.0-70.1)$ & 0.02 \\
\hline RVESVI $\left(\mathrm{ml} / \mathrm{m}^{2}\right)$ & $59.1(47.7-80.9)$ & $35.0(23.0-37.4)$ & $29.8(26.8-32.4)$ & 0.007 \\
\hline RV T1 pre-contrast (ms) & $1056(1021-1081)$ & $1003(922-1029)$ & $974(927-996)$ & 0.005 \\
\hline RV T1 post-contrast (ms) & $482(428-509)$ & $480(449-506)$ & $514(503-538)$ & 0.05 \\
\hline RV-ECV & $0.343(0.331-0.352)$ & $0.294(0.272-0.301)$ & $0.270(0.251-0.281)$ & $<0.0001$ \\
\hline LVEF (\%) & $55.0(50.0-58.5)$ & $28.0(19.0-31.0)$ & $61.2(59.7-62.9)$ & $<0.0001$ \\
\hline LVEDVI $\left(\mathrm{ml} / \mathrm{m}^{2}\right)$ & $58.2(43.1-66.1)$ & 109.0 (102.9-124.8) & $84.3(80.3-90.3)$ & 0.0001 \\
\hline LVESVI $\left(\mathrm{ml} / \mathrm{m}^{2}\right)$ & $28.2(21.2-41.6)$ & $62.3(45.5-79.6)$ & $32.7(29.9-37.4)$ & 0.001 \\
\hline LVMI $\left(\mathrm{g} / \mathrm{m}^{2}\right)$ & $36.9(32.7-41.0)$ & $61.1(50.7-70.5)$ & $32.3(30.1-35.8)$ & 0.008 \\
\hline LV T1 pre-contrast (ms) & $988(963-1006)$ & $969(921-988)$ & $971(948-986)$ & 0.40 \\
\hline LV T1 post-contrast (ms) & $495(438-542)$ & $480(454-505)$ & $519(491-546)$ & 0.28 \\
\hline LV-ECV & $0.305(0.295-0.308)$ & $0.276(0.258-0.290)$ & $0.271(0.251-0.279)$ & 0.002 \\
\hline
\end{tabular}

Results are shown as the median (IQR)

ECV extracellular volume fraction, LV left ventricle, LVEDVI left ventricular end-diastolic volume index, LVEF left ventricular ejection fraction, $L V E S V I$ left ventricular end-systolic volume index, $R V$ right ventricle, RVEDVI right ventricular end-diastolic volume index, RVEF right ventricular ejection fraction, $R V E S V I$ right ventricular end-systolic volume index 

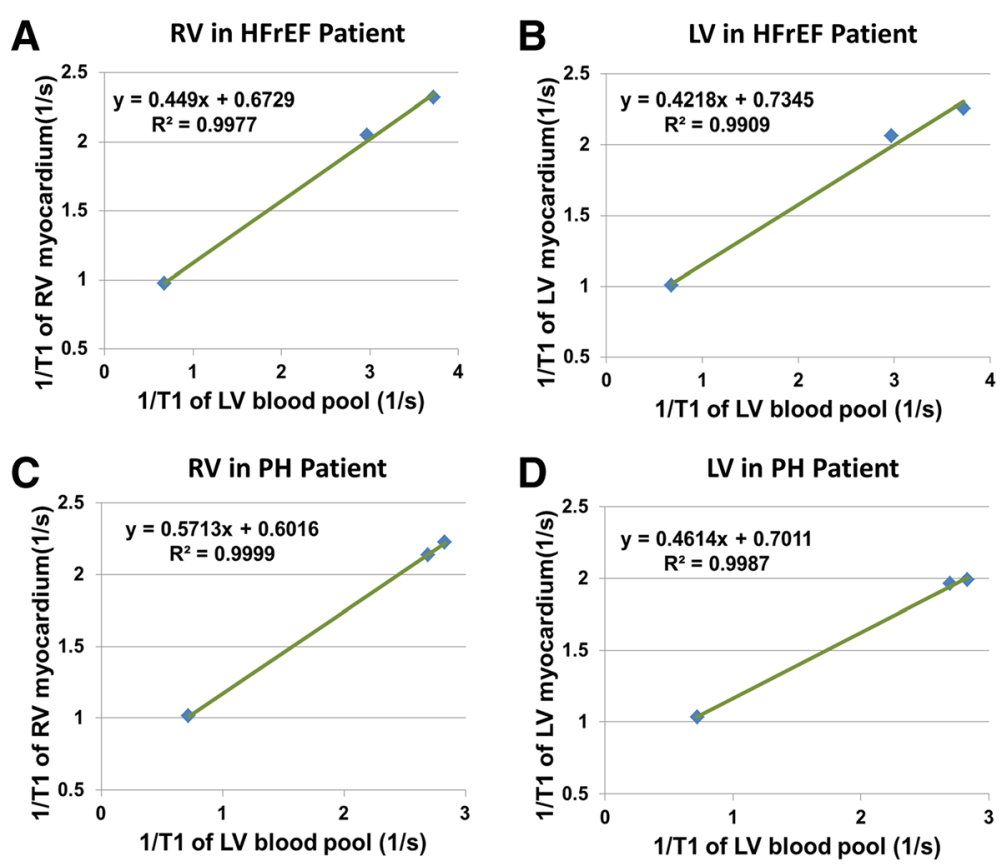

Fig. 2 Myocardial partition coefficients for gadolinium-DTPA ( $\lambda_{\text {Gadolinium }}$ ) in patients with PH and HFrEF. Results for the right ventricular (RV) and left ventricular (LV) $\lambda_{\text {Gadolinium }}$ are shown for patients with heart failure with reduced ejection fraction (HFrEF) (a, $\left.\mathbf{b}\right)$ and pulmonary hypertension (PH) $(\mathbf{c}, \mathbf{d}) . \lambda_{\text {Gadolinium }}$ is estimated as the slope of the linear fit of 1/(myocardial T1) vs $1 /$ (LV blood pool T1) measured at various time points pre- and post-injection of gadolinium-DTPA

Comparison of RV-ECV in $\mathrm{PH}, \mathrm{HFrEF}$, and normal volunteers Figure 4a summarizes the ranges for RV-ECV among patients with $\mathrm{PH}, \mathrm{HFrEF}$, and normal volunteers. The RV-ECV in $\mathrm{PH}$ patients was $27.2 \%$ greater than the RV-ECV in normal volunteers (0.341 v. 0.268; $p<0.0001)$ and $18.9 \%$ greater than the RV-ECV in HFrEF patients (0.341 v. $0.287 ; p<0.0001)$. RV-ECV was greater than LV-ECV in $\mathrm{PH}$ (RV-LV difference $=$ $0.04)$, but RV-ECV was nearly equivalent to LV-ECV in normal volunteers (RV-LV difference $=0.002)(p<$ 0.0001 for RV-LV difference in $\mathrm{PH}$ versus normal volunteers).

\section{Independence of RV-ECV from RVEF and detection of occult RV fibrosis}

In all study participants from all groups, greater RVECV by ANGIE was associated with decreasing RVEF by CMR $(r=-0.75, p=0.001)$. In Fig. 5, patients were grouped into three RVEF groups and three RV-ECV groups. A normal RV-ECV was defined as less than $28.7 \%$ based on the RV-ECV value at the 99th percentile for RV-ECV in the 10 normal volunteers (range 0.249-0.287). Normal LV-ECV values have also been published for patients with T1 mapping in $1.5 \mathrm{~T}$ and $3.0 \mathrm{~T}$ magnets [26, 27]; however, we used data from our normal volunteers for the normal RV-ECV values, which are not well-established in the literature. Among the 22 patients in the $\mathrm{PH}$ and HFrEF groups, forty-three percent of patients with a normal RVEF had an abnormal RV-ECV (ECV Group 2), indicating a significant prevalence of occult fibrosis despite a normal RVEF. In patients with mildly depressed RVEF, $50 \%$ of the patients had an elevated RV-ECV (ECV Group 2). In patients with a moderately-severely depressed RVEF, there was a range of abnormal ECV, with $43 \%$ having a modest increase in RV-ECV (ECV Group 2), and $57 \%$ having a more severe increase in RV-ECV. These ranges demonstrate that patients with similar degrees of RV dysfunction by RVEF can have variable RV-ECV values (ECV group 3). In fact, RVECV ranged from $0.25-0.37$ in patients with an abnormal RVEF.

\section{Multivariable model: RV-ECV, RVEF, and RV end-diastolic volume}

Figure 6 summarizes the complex relationship between RV-ECV, RVEDVI, and RVEF. The multivariable linear model with these variables is described in more detail in Table 3. Both decreasing RVEF and increasing RVEDVI were independently associated with increasing RV-ECV in these patients. Even after adjustment for RVEF and RVEDVI, the clinical diagnostic group ( $\mathrm{PH} \mathrm{v}$. HFrEF v. Normal) was still independently associated with RV-ECV. The overall $\mathrm{R}^{2}$ for this model was 0.804 (adjusted $\mathrm{R}^{2}=0.783 ; p<0.0001$ ). The independent association of RV-ECV with the diagnostic group even 


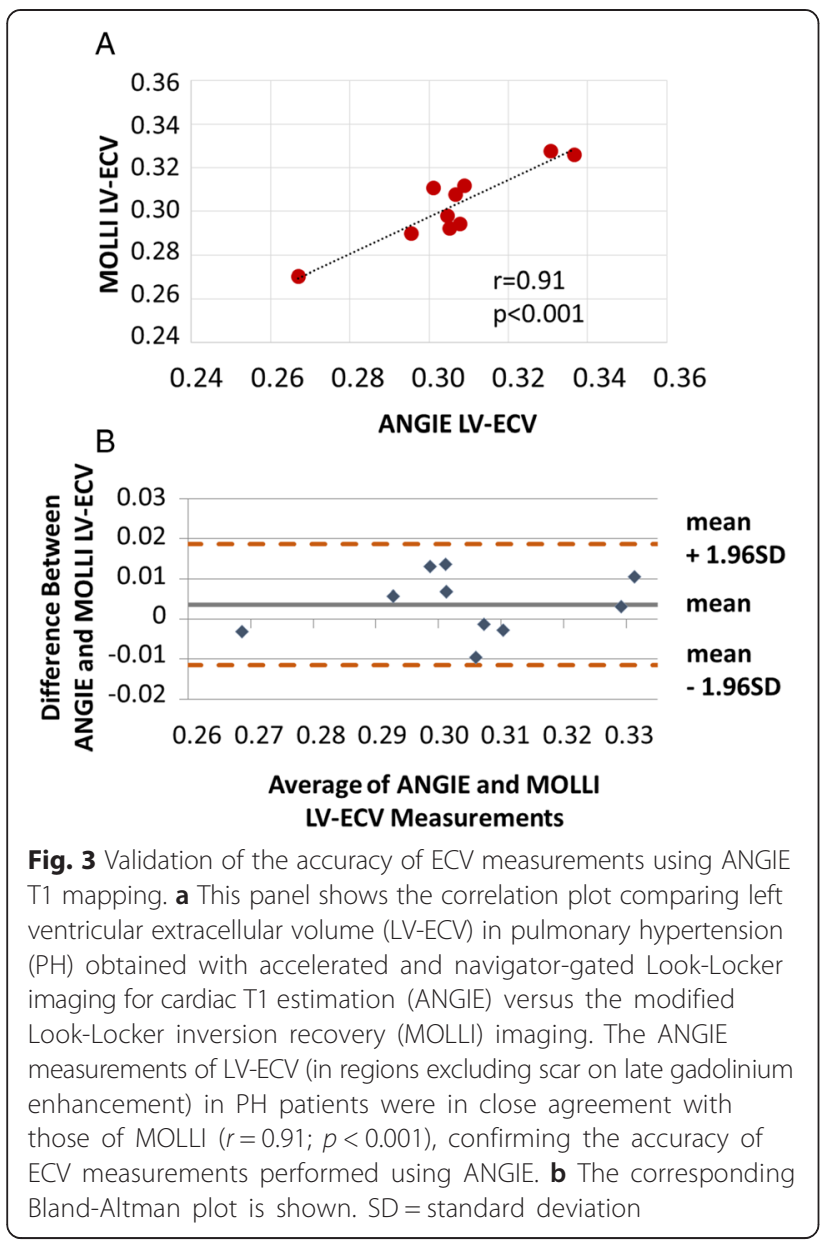

after adjustment for RVEF and RVEDVI suggests the presence of independent factors associated with $\mathrm{PH}$ relative to other groups that lead to increased ECV independent of the degree of RV dilatation or dysfunction.

\section{Interobserver variability}

We also assessed interobserver variability for RV-ECV and RV T1 values before and after contrast in 10 randomly selected participants with contrast-enhanced MRIs in the PH and HFrEF groups. We found that the ICC for RV-ECV was 0.851, the ICC for the RV pre-contrast T1 value was 0.945 , and the ICC for the RV post-contrast T1 value was 0.798 .

\section{Discussion}

This is the first report of $\mathrm{T} 1$ and ECV determination for the RV in a clinical population of patients with $\mathrm{PH}$ or HFrEF without PH. There were several key clinical findings of particular importance to the field of CMR, T1 mapping, RV imaging, ECV determination, and PH. First, ANGIE ECV assessment for the RV is feasible in these patient groups and generates high- resolution results for both the RV and the LV. Second,
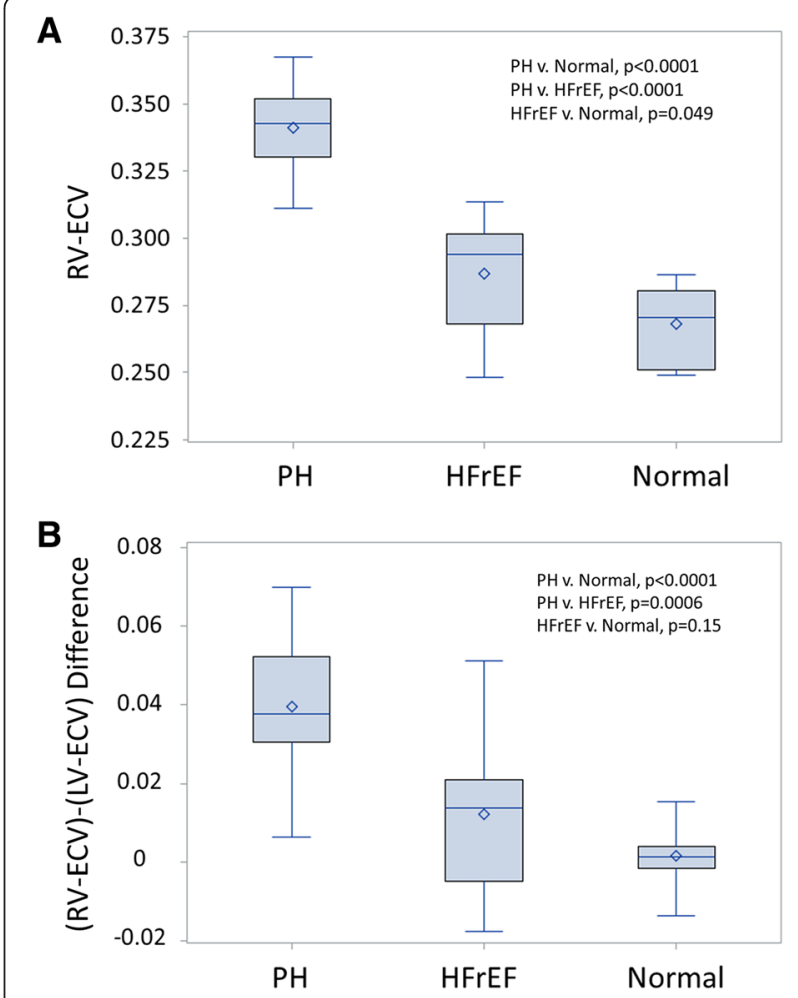

Fig. 4 ECV results in $\mathrm{PH}, \mathrm{HFrEF}$, and normal volunteers. a Box plots are shown for these three groups of patients. RV-ECV is higher in PH versus $\mathrm{V}$ HFrEF ( $p<0.0001)$, in PH versus normal volunteers $(p<0.0001)$, and in HFrEF versus normal volunteers $(p=0.049)$. b Box plots are shown for the differences in RV-ECV and LV-ECV in these three groups of patients. The difference between RV-ECV and LV-ECV is greater in PH versus HFrEF $(p<0.0006)$ and greater in PH versus normal volunteers $(p<0.0001)$. The RV-LV ECV difference in HFrEF versus normal volunteers did not meet statistical significance $(p=0.15)$

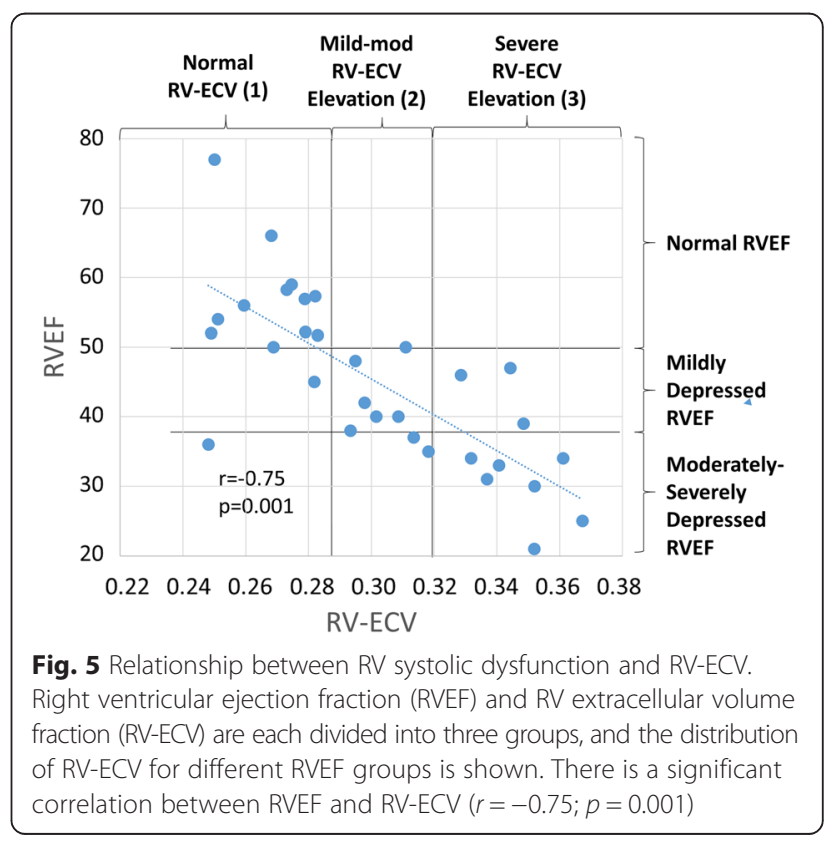




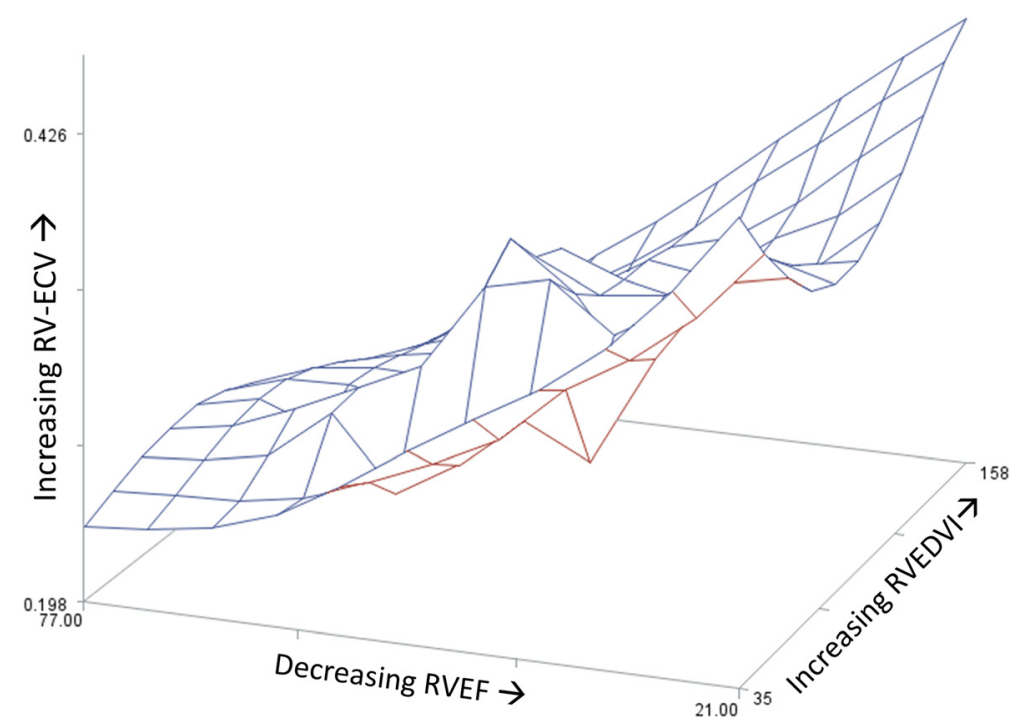

Fig. 6 3D depiction of relationships among RV Fibrosis, dysfunction, and dilatation. The 3D surface plot shows the complex relationships among RV-ECV, RVEF, and RVEDVI. Please also refer to Table 3, which shows that patient group, RVEDVI, and RVEF are independently associated with RV-ECV. The surface is highest in the corner corresponding to decreased RVEF and increased RVEDVI

ANGIE measurements of LV-ECV correlated well with MOLLI measurements of LV-ECV. Although MOLLI measurements of RV T1 values were of insufficient resolution to permit comparison with the higher resolution ANGIE T1 and ECV measurements, the strong correlation between MOLLI and ANGIE for LV-ECV is very important because it shows for the first time that ECV values of the LV as measured with ANGIE are comparable to those determined with MOLLI imaging.

The third significant finding is that ANGIE detected significantly higher RV-ECV values in $\mathrm{PH}$ patients compared with normal volunteers and patients with HFrEF without $\mathrm{PH}$. Of note, although RV involvement in HFrEF is not uncommon, particularly those with $\mathrm{PH}$, the patients we chose for the HFrEF cohort did not have $\mathrm{PH}$. In addition, the measures of RV size and function were closer to those of normal volunteers in patients with HFrEF compared with the corresponding measures in patients in the $\mathrm{PH}$ group. In particular, RVEDVI was reduced by more than $30 \%$ in HFrEF, while RVESVI was reduced by more than $40 \%$ in HFrEF compared with $\mathrm{PH}$.

A fourth significant finding is that RV-ECV represents a unique parameter describing RV structure that is associated with RVEF and RVEDVI but is not merely a surrogate for one of these parameters. This was shown by the results of the multivariable linear model with RV-ECV as the outcome variable. In this model, RV-ECV was independently associated with both RVEF and RVEDVI modeled as independent variables. Furthermore, the clinical diagnostic group was also highly associated with RV-ECV even after adjustment for RVEF and RVEDVI. This is consistent with independent biologic factors in $\mathrm{PH}$ that result in greater RV-ECV in $\mathrm{PH}$ compared with patients having HFrEF and normal volunteers, independent of the degree of RV dysfunction and dilatation.

\section{Imaging of fibrosis and scar in the RV in pulmonary hypertension}

The findings justify further study regarding the use of this RV fibrosis imaging technique in patients with $\mathrm{PH}$ [3-6]. While noninvasive imaging methods have shown several measurable indices to be reliable surrogates for RV function (such as RV strain [11]), there has not been an effective way to image diffuse RV fibrosis prior to the present study. While the use of LGE can be used to localize focal fibrosis [12, 28], its

Table 3 Multivariable model for RV-ECV

\begin{tabular}{llllll}
\hline Variable & DF & Parameter estimate & Standard error & t value & $p$ value \\
\hline Intercept & 1 & 0.361 & 0.0182 & 19.8 & 0.0001 \\
RVEF & 1 & -0.000829 & 0.000360 & -2.3 & 0.03 \\
RVEDVI & 1 & 0.000296 & 0.000128 & 2.32 & 0.03 \\
Diagnostic group & 1 & -0.0234 & 0.00563 & -4.16 & 0.0003 \\
\hline
\end{tabular}


use in patients with $\mathrm{PH}$ has been limited to imaging of LV septal fibrosis at the RV insertion points [29, 30]. Although it is clear that fibrosis is important in the $\mathrm{RV}$ in $\mathrm{PH}$, traditional imaging of scar with LGE in the RV free wall is technically difficult. Furthermore, while T1 mapping protocols such as MOLLI have been used to quantify the myocardial ECV in the LV, their use in the RV has been limited because of inadequate spatial resolution.

\section{ANGIE as a solution to the inherent challenges of RV T1 mapping}

ANGIE presents a unique solution to T1 mapping of the thin RV free wall. Just as we showed the high quality of native ANGIE imaging of the RV in a recent publication [20], the high quality of post-contrast ANGIE T1 mapping of the RV is shown in Fig. 1. This high resolution is achieved by several innovative imaging techniques, described previously. Briefly, ANGIE employs navigator gating to allow acquisition of images during free breathing and, consequently, to achieve higher spatial resolution. To do so in a reasonable scan time, ANGIE also uses compressed sensing and parallel imaging for acceleration. These innovations provide high resolution imaging of the thin-walled RV in a very acceptable period of imaging time. ANGIE results for both the RV and LV can be obtained during a single study and using the same contrast injection used for LGE imaging of the LV, also performed during the study. As described in the methods, one pre-contrast native ANGIE acquisition and two post-LGE ANGIE acquisitions are typically performed. Analysis of RV-ECV was found to be very reproducible. The ICC of 0.945 for interobserver agreement for pre-contrast RV T1 values was also quite high and consistent with values obtained in volunteers in our previously published study using this technique [20]. The ICC for post-contrast RV T1 values was also very good but slightly lower than that for the RV pre-contrast T1 values.

\section{Clinical applications and significance}

There are a number of potential clinical applications of RV fibrosis assessment in patients with $\mathrm{PH}$ and others with HFrEF. First, RV-ECV could be used for risk stratification of patients with $\mathrm{PH}$ in combination with RVEF, if additional follow-up data confirm that RV-ECV, like RVEF, is associated with adverse clinical outcomes. Second, RV-ECV assessment could be used to monitor the effectiveness of various therapies [31-33] for $\mathrm{PH}$ by performing longitudinal CMR assessments with RVECV measurements. Third, RV-ECV assessment could be used in animal models of $\mathrm{PH}$ in the context of drug development to compare how novel therapeutic agents modify the effect of PH on RV fibrosis. Fourth,
RV-ECV mapping could be used to evaluate RV reserve in patients being evaluated for cardiac surgery, pulmonary endartectomy, or lung transplantation, as early postoperative right ventricular failure is a significant cause of morbidity and mortality in these patients.

\section{Limitations}

While the sample size of the present study is adequate to assess the feasibility and high quality of this method for RV-ECV determination, as well associations between RV-ECV and other patient findings, future studies with ANGIE in $\mathrm{PH}$ to evaluate associations with clinical events are indicated but would require a larger number of patients. We also acknowledge that fat suppression could be a potential contaminant of RV T1 and ECV measurements. Of note, we used fat suppression for the purpose of limiting partial volume contamination by fat; however, ANGIE images can be acquired without fat suppression. Although MRI ECV measurements have been found to correlate with histologic fibrosis, and we interpreted elevated ECV in this study as a surrogate for fibrosis, inflammation and edema may also contribute to an increased ECV. There were differences in gender in the groups, but both the $\mathrm{PH}$ and volunteer group were similar in the sense that both had more females than males. Volunteers were younger than PH and HFrEF patients, but it has recently been demonstrated that age does not have a significant effect on CMR measurements of ECV [34]. Another limitation is that ANGIE was applied to only two slices. To overcome the latter limitation, we are developing 3D ANGIE methods for whole heart coverage and more efficient RV imaging in the future [35].

\section{Conclusions}

Pre- and post-contrast ANGIE imaging provides highresolution T1 mapping and ECV assessments for not only the LV but also the RV, with LV-ECV by ANGIE and MOLLI in close agreement. Elevated RV-ECV in $\mathrm{PH}$ is independently associated not only with RV chamber dilatation and dysfunction, but also with the clinical diagnostic group, suggesting that increased RV-ECV/RV fibrosis in $\mathrm{PH}$ results from biologic factors that cannot be predicted by volumetric RV findings on CMR. This highlights the importance of assessing RV-ECV in addition to RV volumes and EF in $\mathrm{PH}$ and other conditions that may adversely affect the RV. Further studies of RV-ECV in PH and HFrEF are indicated to assess associations among RV-ECV, functional limitations, and long-term clinical outcomes, as well as the effects of drug therapy for $\mathrm{PH}$ on RV-ECV. 


\section{Abbreviations}

ANGIE: Accelerated and navigator-gated Look-Locker imaging for cardiac T1 estimation; CMR: Cardiovascular magnetic resonance; CTEPH: Chronic thromboembolic pulmonary hypertension; DTPA: Diethylene triamine pentaacetic acid; ERA: Endothelin receptor antagonists; FOV: Field of view; HFrEF: Heart failure with reduced ejection fraction; $\lambda$-Gd: Partition coefficient of gadolinium; LGE: Late gadolinium enhancement; LV-ECV: Left ventricular extracellular volume fraction; LVEDVI: Left ventricular end-diastolic volume index; LVEF: Left ventricular ejection fraction; LVESVI: Left ventricular end-systolic volume index; MOLLI: Modified Look-Locker inversion recovery; $\mathrm{PH}$ : Pulmonary hypertension; RV-ECV: Right ventricular extracellular volume fraction; RVEDVI: Right ventricular end-diastolic volume index; RVEF: Right ventricular ejection fraction; RVESVI: Right ventricular end-systolic volume index; TE: Echo time; TR: Repetition time; WHO: World Health Organization.

\section{Competing interests}

Dr. Epstein and Dr. Kramer have received research grant support from Siemens Healthcare. Dr. Chen is now employed by Siemens Healthcare, although he was a PhD student at the University of Virginia at the time of this research.

\section{Authors' contributions}

All authors: 1) have made substantial contributions to conception and design, or acquisition of data, or analysis and interpretation of data; 2) have been involved in drafting the manuscript or revising it critically for important intellectual content; 3) have given final approval of the version to be published; and 4) agree to be accountable for all aspects of the work in ensuring that questions related to the accuracy or integrity of any part of the work are appropriately investigated and resolved. The following specific contributions were made by each author: BM: Developed ANGIE sequence, performed MRI scans, analyzed data, drafted manuscript. DA: Performed MRI scans, analyzed MRI data, critically revised manuscript. JG: Performed MRI scans, analyzed MRI data, critically revised manuscript. XC: Performed MRI scans, analyzed MRI data, critically revised manuscript. W: Recruited patients, analyzed MRI data, critically revised manuscript. KC: Performed MRI scans, analyzed MRI data, critically revised manuscript. CS: Analyzed MRI data, critically revised manuscript. SM: Recruited patients, helped conceive study, critically revised manuscript. JK: Recruited patients, helped conceive study, critically revised manuscript. EG: Recruited patients, helped conceive study, critically revised manuscript. MS: Involved in sequence development, analyzed MRI data, critically revised manuscript. CK: Conceived study, critically revised manuscript. FE: Conceived study, developed MRI sequence, analyzed MRI data, critically revised manuscript. KB: Conceived study, recruited subjects, drafted manuscript.

\section{Acknowledgements}

We appreciate the help of our MRI technologists, Joe Hylton and John Christopher, in performing the scans. We also appreciate the work of our research coordinator, Hollis Phillips, in helping to coordinate the participant visits for this study.

\section{Funding}

The study was funded by the following grants: NIH K23HL094761 (Bilchick), NIH R01EB001763 (Epstein), and AHA 14PRE20210008 (Mehta).

\section{Author details}

${ }^{1}$ Department of Medicine, University of Virginia Health System, P.O. Box 800158, Charlottesville, VA 22908, USA. ²Department of Biomedical Engineering, University of Virginia Health System, Charlottesville, VA, USA. ${ }^{3}$ Department of Radiology, University of Virginia Health System, Charlottesville, VA, USA.

\section{Received: 5 July 2015 Accepted: 19 November 2015}

Published online: 21 December 2015

\section{References}

1. Chen EP, Craig DM, Bittner HB, Davis RD, Van TP. Pharmacological strategies for improving diastolic dysfunction in the setting of chronic pulmonary hypertension. Circulation. 1998;97:1606-12.
2. Louie EK, Lin SS, Reynertson SI, Brundage BH, Levitsky S, Rich S. Pressure and volume loading of the right ventricle have opposite effects on left ventricular ejection fraction. Circulation. 1995;92:819-24.

3. D'Alonzo GE, Barst RJ, Ayres SM, Bergofsky EH, Brundage BH, Detre KM, et al. Survival in patients with primary pulmonary hypertension. Results from a national prospective registry. Ann Intern Med. 1991;115:343-9.

4. McLaughlin W, Sitbon O, Badesch DB, Barst RJ, Black C, Galie N, et al. Survival with first-line bosentan in patients with primary pulmonary hypertension. Eur Respir J. 2005;25:244-9.

5. Sandoval J, Bauerle O, Palomar A, Gomez A, Martinez-Guerra ML, Beltran M, et al. Survival in primary pulmonary hypertension. Validation of a prognostic equation. Circulation. 1994;89:1733-44.

6. Chin KM, Kim NH, Rubin LJ. The right ventricle in pulmonary hypertension. Coron Artery Dis. 2005:16:13-8.

7. Haddad F, Doyle R, Murphy DJ, Hunt SA. Right ventricular function in cardiovascular disease, part II: pathophysiology, clinical importance, and management of right ventricular failure. Circulation. 2008;117:1717-31.

8. Guazzi M, Borlaug BA. Pulmonary hypertension due to left heart disease. Circulation. 2012;126:975-90.

9. Moraes DL, Colucci WS, Givertz MM. Secondary pulmonary hypertension in chronic heart failure: the role of the endothelium in pathophysiology and management. Circulation. 2000;102:1718-23.

10. Ichihara S, Senbonmatsu T, Price Jr E, Ichiki T, Gaffney FA, Inagami T. Angiotensin II type 2 receptor is essential for left ventricular hypertrophy and cardiac fibrosis in chronic angiotensin II-induced hypertension. Circulation. 2001;104:346-51.

11. Rudski LG, Lai WW, Afilalo J, Hua L, Handschumacher MD, Chandrasekaran K, et al. Guidelines for the echocardiographic assessment of the right heart in adults: a report from the American Society of Echocardiography endorsed by the European Association of Echocardiography, a registered branch of the European Society of Cardiology, and the Canadian Society of Echocardiography. J Am Soc Echocardiogr. 2010;23:685-713.

12. Schmidt A, Azevedo CF, Cheng A, Gupta SN, Bluemke DA, Foo TK, et al. Infarct tissue heterogeneity by magnetic resonance imaging identifies enhanced cardiac arrhythmia susceptibility in patients with left ventricular dysfunction. Circulation. 2007;115:2006-14.

13. Fernandes VR, Wu KC, Rosen BD, Schmidt A, Lardo AC, Osman N, et al. Enhanced infarct border zone function and altered mechanical activation predict inducibility of monomorphic ventricular tachycardia in patients with ischemic cardiomyopathy. Radiology. 2007;245:712-9.

14. Messroghli DR, Radjenovic A, Kozerke S, Higgins DM, Sivananthan MU, Ridgway JP. Modified Look-Locker inversion recovery (MOLLI) for highresolution T1 mapping of the heart. Magn Reson Med. 2004;52:141-6.

15. Messroghli DR, Greiser A, Frohlich M, Dietz R, Schulz-Menger J. Optimization and validation of a fully-integrated pulse sequence for modified look-locker inversionrecovery (MOLLI) T1 mapping of the heart. J Magn Reson Imaging. 2007;26:1081-6.

16. Messroghli DR, Walters K, Plein S, Sparrow P, Friedrich MG, Ridgway JP, et al. Myocardial T1 mapping: application to patients with acute and chronic myocardial infarction. Magn Reson Med. 2007:58:34-40.

17. Iles L, Pfluger H, Phrommintikul A, Cherayath J, Aksit P, Gupta SN, et al. Evaluation of diffuse myocardial fibrosis in heart failure with cardiac magnetic resonance contrast-enhanced T1 mapping. J Am Coll Cardiol. 2008;52:1574-80.

18. Fontana M, White SK, Banypersad SM, Sado DM, Maestrini V, Flett AS, et al. Comparison of T1 mapping techniques for ECV quantification. Histological validation and reproducibility of ShMOLLI versus multibreath-hold T1 quantification equilibrium contrast CMR. J Cardiovasc Magn Reson. 2012;14:88.

19. Stuckey DJ, MCSweeney SJ, Thin MZ, Habib J, Price AN, Fiedler LR, et al. T1 mapping detects pharmacological retardation of diffuse cardiac fibrosis in mouse pressure-overload hypertrophy. Circ Cardiovasc Imaging. 2014;7(2):240-9.

20. Mehta BB, Chen X, Bilchick KC, Salerno M, Epstein FH. Accelerated and navigator-gated look-locker imaging for cardiac t1 estimation (ANGIE): development and application to T1 mapping of the right ventricle. Magn Reson Med. 2015;73:150-60.

21. Mehta B, Gonzalez J, Salerno M, Workman V, Mazimba S, Kennedy J, et al. High-resolution T1 mapping with ANGIE detects increased right-ventricular extracellular volume fraction in patients with pulmonary hypertension (abstr). J Cardiovasc Magn Reson. 2015;17 Suppl 1:039.

22. Kuruvilla S, Janardhanan R, Antkowiak P, Keeley EC, Adenaw N, Brooks J, et al. Increased extracellular volume and altered mechanics are associated with LVH in hypertensive heart disease, not hypertension alone. JACC Cardiovasc Imaging 2015:8:172-80 
23. Salerno M, Janardhanan R, Jiji RS, Brooks J, Adenaw N, Mehta B, et al. Comparison of methods for determining the partition coefficient of gadolinium in the myocardium using T1 mapping. J Magn Reson Imaging. 2013;38:217-24.

24. Jerosch-Herold M, Sheridan DC, Kushner JD, Nauman D, Burgess D, Dutton D, et al. Cardiac magnetic resonance imaging of myocardial contrast uptake and blood flow in patients affected with idiopathic or familial dilated cardiomyopathy. Am J Physiol Heart Circ Physiol. 2008:295:H1234-42.

25. Kehr E, Sono M, Chugh SS, Jerosch-Herold M. Gadolinium-enhanced magnetic resonance imaging for detection and quantification of fibrosis in human myocardium in vitro. Int J Cardiovasc Imaging. 2008;24:61-8.

26. Florian A, Ludwig A, Rosch S, Yildiz H, Sechtem U, Yilmaz A. Myocardial fibrosis imaging based on T1-mapping and extracellular volume fraction (ECV) measurement in muscular dystrophy patients: diagnostic value compared with conventional late gadolinium enhancement (LGE) imaging. Eur Heart J Cardiovasc Imaging. 2014;15:1004-12.

27. Puntmann VO, Voigt T, Chen Z, Mayr M, Karim R, Rhode K, et al. Native T1 mapping in differentiation of normal myocardium from diffuse disease in hypertrophic and dilated cardiomyopathy. JACC Cardiovasc Imaging. 2013;6:475-84.

28. Kim RJ, Wu E, Rafael A, Chen EL, Parker MA, Simonetti O, et al. The use of contrast-enhanced magnetic resonance imaging to identify reversible myocardial dysfunction.[see comment]. N Engl J Med. 2000;343:1445-53.

29. Shehata ML, Lossnitzer D, Skrok J, Boyce D, Lechtzin N, Mathai SC, et al. Myocardial delayed enhancement in pulmonary hypertension: pulmonary hemodynamics, right ventricular function, and remodeling. AJR Am J Roentgenol. 2011;196:87-94.

30. Freed BH, Gomberg-Maitland M, Chandra S, Mor-Avi V, Rich S, Archer SL, et al. Late gadolinium enhancement cardiovascular magnetic resonance predicts clinical worsening in patients with pulmonary hypertension. J Cardiovasc Magn Reson. 2012;14:11.

31. Oudiz RJ, Roveran G, Hansen JE, Sun XG, Wasserman K. Effect of sildenafi on ventilatory efficiency and exercise tolerance in pulmonary hypertension. Eur J Heart Fail. 2007:9:917-21.

32. Barst RJ, McGoon M, McLaughlin V, Tapson V, Rich S, Rubin L, et al. Beraprost therapy for pulmonary arterial hypertension. J Am Coll Cardiol. 2003;41:2119-25.

33. Wensel R, Opitz CF, Ewert R, Bruch L, Kleber FX. Effects of iloprost inhalation on exercise capacity and ventilatory efficiency in patients with primary pulmonary hypertension. Circulation. 2000;101:2388-92.

34. Dabir D, Child N, Kalra A, Rogers T, Gebker R, Jabbour A, et al. Reference values for healthy human myocardium using a T1 mapping methodology: results from the international $\mathrm{T} 1$ multicenter cardiovascular magnetic resonance study. J Cardiovasc Magn Reson. 2014;16:69.

35. Mehta BB, Salerno M, Epstein FH. High-resolution three-dimensional ANGIE T1 mapping of the whole heart (abstr). J Cardiovasc Magn Reson. 2015;17:1-3.

\section{Submit your next manuscript to BioMed Central and we will help you at every step:}

- We accept pre-submission inquiries

- Our selector tool helps you to find the most relevant journal

- We provide round the clock customer support

- Convenient online submission

- Thorough peer review

- Inclusion in PubMed and all major indexing services

- Maximum visibility for your research

Submit your manuscript at www.biomedcentral.com/submit

O Biomed Central 